\title{
Quantum Message Exchange Based on Entanglement and Bell-State Measurements
}

\author{
Sung Soon Jang and Hai-Woong Lee \\ Department of Physics, Korea Advanced Institute of Science and Technology \\ Daejeon, 305-701, Korea
}

\begin{abstract}
We propose a scheme by which two parties can secretely and simultaneously exchange messages. The scheme requires the two parties to share entanglement and both to perform Bell-state measurements. Only two out of the four Bell states are required to be distinguished in the Bell-state measurements, and thus the scheme is experimentally feasible using only linear optical means. Generalizations of the scheme to high-dimensional systems and to multipartite entanglement are considered. We show also that the proposed scheme works even if the two parties do not possess shared reference frames.
\end{abstract}

\section{Introduction}

Entanglement is an essential resource for many applications in quantum information science such as quantum superdense coding[1,2] quantum teleportation $[3,4,5,6,7,8]$, quantum cryptography[9,10,11], and quantum computing[12,13]. From an informationtheoretic point of view, two parties sharing entanglement can be regarded to have already a certain amount of information distributed between them; one e-bit per a shared maximally entangled pair of qubits. Thus, for example, in superdense coding two bits of information can be sent from one party to 
another by manipulating only one of two maximally entangled qubits. In quantum teleportation a quantum state of a qubit can be completely transferred by sending only two bits of classical information, if the two parties, the sender and the receiver, share a maximally entangled pair.

In this work we explore yet another situation in which two(or more) parties can make use of entanglement they share to their advantage. We consider a situation in which two parties, Alice and Bob, share a maximally entangled pair A and B of qubits. Alice makes a Bell-state measurement upon the qubit $A$ and another qubit $\alpha$ she prepared in a state about which only she has the information. Bob also makes a Bell-state measurement upon the qubit $B$ and another qubit $\beta$ he prepared in a state about which only he has the information. We are interested in the probability for each of the sixteen possible joint measurement outcomes, which in general depends upon the states of the qubits $\alpha$ and $\beta$ in a way characteristic of the shared entanglement. If Alice keeps the information on the state of qubit $\alpha$ to herself and Bob keeps the information on the state of qubit $\beta$ to himself, they have a partial knowledge of the probabilities in advance that others do not. We suggest that this advantage can be exploited to devise a method by which Alice and Bob secretely and simultaneously exchange messages. Generalizations of the method to higher-dimensional systems( "qudits") and to multipartite entanglement are also discussed.

\section{The Basic Scheme}

Let us suppose that two parties, Alice and Bob, share a maximally entangled pair $A$ and $B$ of qubits. The qubits $\mathrm{A}$ and $\mathrm{B}$ can be in any of the four Bell states

$$
\left|\Phi_{i j}\right\rangle_{A B}=\frac{1}{\sqrt{2}} \sum_{q=0}^{1}(-1)^{j q}|q\rangle_{A}|q+i\rangle_{B} ; i, j=0,1
$$




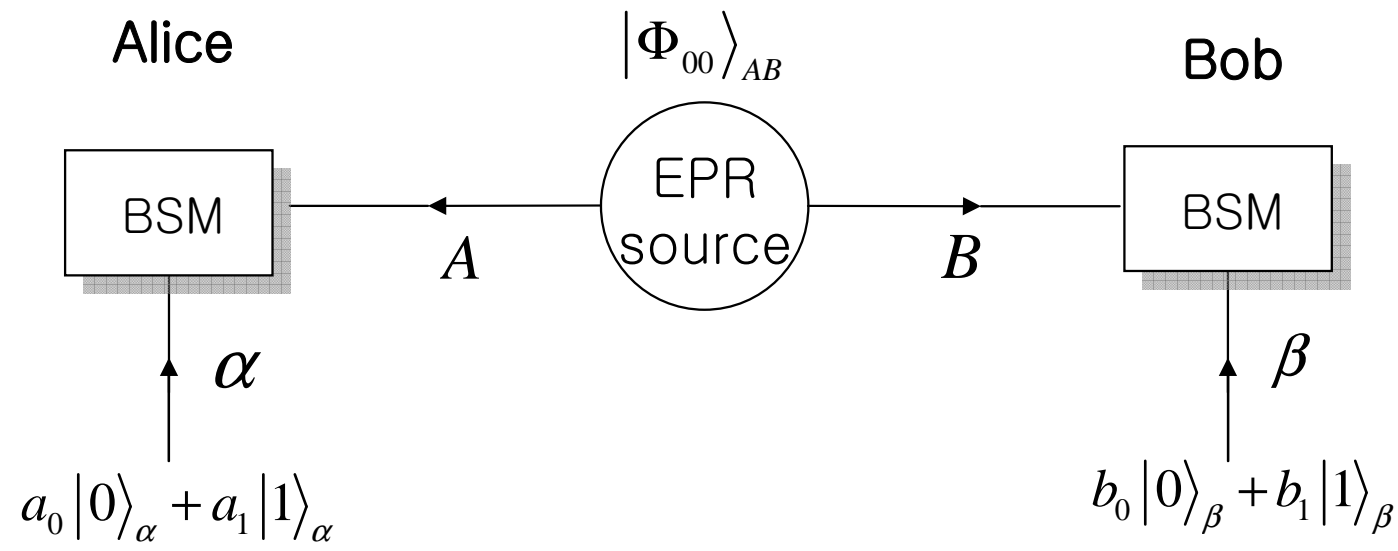

Fig. 1. Experimental Scheme. The EPR(Einstein-Podolsky-Rosen) source emits an entangled pair in state $\left|\Phi_{00}\right\rangle_{A B}$. Alice performs a Bell-state measurement on the qubit pair $\alpha$ and $\mathrm{A}$, and Bob performs a Bell-state measurement on the qubit pair $\beta$ and B. BSM stands for Bell-state measurement.

but for the sake of concreteness of argument, we take it as

$$
\left|\Phi_{00}\right\rangle_{A B}=\frac{1}{\sqrt{2}}\left(|0\rangle_{A}|0\rangle_{B}+|1\rangle_{A}|1\rangle_{B}\right)
$$

Alice has in her possession another qubit $\alpha$ which she prepared in state

$$
|\psi\rangle_{\alpha}=a_{0}|0\rangle_{\alpha}+a_{1}|1\rangle_{\alpha}
$$

Similarly, Bob has in his possession yet another qubit $\beta$ which he prepared in state

$$
|\psi\rangle_{\beta}=b_{0}|0\rangle_{\beta}+b_{1}|1\rangle_{\beta}
$$

Now Alice performs a Bell-state measurement on the pair $\alpha$ and $A$, and Bob performs a Bell-state measurement on the pair $\beta$ and $B$. The experimental scheme is depicted schematically in Fig. 1.

The probability $P_{i_{1} j_{1} i_{2} j_{2}}\left(i_{1}, j_{1}, i_{2}, j_{2}=0,1\right)$ that Alice's Bell-state measurement yields $\left|\Phi_{i_{1} j_{1}}\right\rangle_{\alpha A}$ and Bob's Bell-state measurement yields $\left|\Phi_{i_{2} j_{2}}\right\rangle_{\beta B}$ can be obtained by expanding the total wave function $|\psi\rangle_{\alpha \beta A B}=|\psi\rangle_{\alpha}|\psi\rangle_{\beta}\left|\Phi_{00}\right\rangle_{A B}$ as

$$
|\psi\rangle_{\alpha \beta A B}=\sum_{i_{1}, j_{1}, i_{2}, j_{2}=0}^{1}\left|\Phi_{i_{1} j_{1}}\right\rangle_{\alpha A}\left|\Phi_{i_{2} j_{2}}\right\rangle_{\beta B} V_{i_{1} j_{1} i_{2} j_{2}}
$$


A straightforward algebra yields

$$
V_{i_{1} j_{1} i_{2} j_{2}}=\frac{1}{2 \sqrt{2}}(-1)^{\left(i_{1} j_{1}+i_{2} j_{2}\right)}\left[a_{i_{1}} b_{i_{2}}+(-1)^{\left(j_{1}+j_{2}\right)} a_{i_{1}+1} b_{i_{2}+1}\right]
$$

where all indices are evaluated modulo 2 . The probabilities $P_{i_{1} j_{1} i_{2} j_{2}}$ 's are given by $P_{i_{1} j_{1} i_{2} j_{2}}=\left|V_{i_{1} j_{1} i_{2} j_{2}}\right|^{2}$. From Eq.(6) we immediately obtain

$$
\begin{aligned}
& P_{0000}=P_{0101}=P_{1010}=P_{1111}=\frac{1}{8}\left|a_{0} b_{0}+a_{1} b_{1}\right|^{2} \\
& P_{0001}=P_{0100}=P_{1011}=P_{1110}=\frac{1}{8}\left|a_{0} b_{0}-a_{1} b_{1}\right|^{2} \\
& P_{0010}=P_{0111}=P_{1000}=P_{1101}=\frac{1}{8}\left|a_{0} b_{1}+a_{1} b_{0}\right|^{2} \\
& P_{0011}=P_{0110}=P_{1001}=P_{1100}=\frac{1}{8}\left|a_{0} b_{1}-a_{1} b_{0}\right|^{2}
\end{aligned}
$$

We note that, since Alice prepared the state of qubit $\alpha$ and thus knows what $a_{0}$ and $a_{1}$ are, and similarly since Bob prepared the state of qubit $\beta$ and thus knows what $b_{0}$ and $b_{1}$ are, Alice and Bob have a partial prior knowledge of the probabilities $P_{i_{1} j_{1} i_{2} j_{2}}$ 's. We suggest that they can take advantage of this knowledge to secretely exchange messages.

The scheme we propose goes as follows. We suppose that Alice and Bob share a large number $\mathrm{N}(\gg 1)$ of maximally entangled pairs A's and B's. We further suppose that Alice has an equally large number $\mathrm{N}$ of qubits $\alpha$ 's, each of which she prepared in state (3), and that Bob has $\mathrm{N}$ qubits $\beta$ 's, each of which he prepared in state (4). Alice keeps the information on the state of qubits $\alpha$ 's to herself and Bob keeps the information on the state of qubits $\beta$ 's to himself. Alice and Bob then perform a series of $\mathrm{N}$ Bell-state measurements on each pair $\alpha$ and $A$, and $\beta$ and $B$, respectively. They announce publicly their measurement results only when the outcome is either $\Phi_{10}$ or $\Phi_{11}$ (this considerably eases the burden on the Bell-state measurements, because only these two Bell states can be unambiguously distinguished with linear optical means 
$[2,7,14])$. By counting the number $N_{1 j_{1} 1 j_{2}}$ of occurrences for the joint outcome $\left|\Phi_{1 j_{1}}\right\rangle_{\alpha A}\left|\Phi_{1 j_{2}}\right\rangle_{\beta B}$, the probabilities $P_{1 j_{1} j_{2}}$ 's $\left(j_{1}, j_{2}=0,1\right)$ can be determined experimentally as

$$
P_{1 j_{1} 1 j_{2}}^{\exp }=\frac{N_{1 j_{1} 1 j_{2}}}{N}
$$

When the four experimentally determined probabilities $P_{1 j_{1} j_{2}}^{\exp }$ 's $\left(P_{1010}^{\exp }, P_{1111}^{\exp }\right.$, $\left.P_{1011}^{\exp }, P_{1110}^{\exp }\right)$ are substituted for the probabilities $P_{1 j_{1} 1 j_{2}}$ 's of Eqs. (7a) and (7b), we obtain two equations that relate the four constants $a_{0}, a_{1}, b_{0}$ and $b_{1}$. Since Alice knows the values of $a_{0}$ and $a_{1}$, there are only two unknowns $b_{0}$ and $b_{1}$ [ the constants $b_{0}$ and $b_{1}$ are complex numbers, but they are subject to normalization and the overall phase can be ignored] to her. Likewise, there are only two unknowns $a_{0}$ and $a_{1}$ to Bob. To any third party, however, the number of unknowns is four. We can thus conclude that only Alice and Bob can completely determine the four constants $a_{0}, a_{1}, b_{0}$ and $b_{1}$. Let us suppose that Alice and Bob have secret messages they wish to send to each other. If they prepare their messages in the form of two constants, the scheme described above can be used for them to achieve a secret two-way communication. We mention that a scheme which is different from our proposed scheme but allows two parties to simultaneously exchange their messages as our proposed scheme does has recently been proposed[15].

\section{Efficiency}

Let us now estimate the efficiency of the scheme described in the previous section. When a sufficiently large number $N \gg 1$ of Bell-state measurements are made by Alice and Bob each, the number $N_{i_{1} j_{1} i_{2} j_{2}}$ of times the joint outcome $\left|\Phi_{i_{1} j_{1}}\right\rangle_{\alpha A}\left|\Phi_{i_{2} j_{2}}\right\rangle_{\beta B}$ is counted lies within the range defined as[16]

$$
N P_{i_{1} j_{1} i_{2} j_{2}}-\sqrt{2 N P_{i_{1} j_{1} i_{2} j_{2}}\left(1-P_{i_{1} j_{1} i_{2} j_{2}}\right)} \lesssim N_{i_{1} j_{1} i_{2} j_{2}}^{e x p} \lesssim N P_{i_{1} j_{1} i_{2} j_{2}}+\sqrt{2 N P_{i_{1} j_{1} i_{2} j_{2}}\left(1-P_{i_{1} j_{1} i_{2} j_{2}}\right)}
$$


where $P_{i_{1} j_{1} i_{2} j_{2}}$ 's are the exact theoretical probabilities given by Eqs.(7). Thus, the accuracy of the experimentally determined probabilities $P_{i_{1} j_{1} i_{2} j_{2}}^{e x p}=N_{i_{1} j_{1} i_{2} j_{2}}^{e x p} / N$ is limited by

$$
\left|P_{i_{1} j_{1} i_{2} j_{2}}^{e x p}-P_{i_{1} j_{1} i_{2} j_{2}}\right| \lesssim \frac{\sqrt{2 N P_{i_{1} j_{1} i_{2} j_{2}}\left(1-P_{i_{1} j_{1} i_{2} j_{2}}\right)}}{N} \cong \frac{1}{\sqrt{N}}
$$

Eq.(10) is valid as long as Alice and Bob perform each of their Bell-state measurements individually, which we assume here. [If a collective approach is adopted, for example, if Alice performs all N Bell-state measurements before Bob makes any of his measurements and sends the message containing the outcome of all her measurements to Bob, and if Bob, upon receiving Alice's message, performs his Bell-state measurements in an appropriate collective way, it may be possible to obtain a higher accuracy for $P_{i_{1} j_{1} i_{2} j_{2}}^{\exp }$. Further research is needed on the collective approach.] Eq.(10) indicates that, in order to obtain $P_{i_{1} j_{1} i_{2} j_{2}}^{\exp }$ accurate to one decimal point, which would allow Alice and Bob to share four real values $\cos \theta_{A}, \cos \phi_{A}, \cos \theta_{B}, \cos \phi_{B}\left(a_{0}=\cos \theta_{A}, a_{1}=\right.$ $\left.\sin \theta_{A} e^{i \phi_{A}}, b_{0}=\cos \theta_{B}, a_{1}=\sin \theta_{B} e^{i \phi_{B}}\right)$ accurate to one decimal point each, Alice and Bob should perform $\sim 100$ (perhaps a few hundred) Bell-state measurements each. We therefore conclude that Alice and Bob gain 4 secret digits(or equivalently 13 14 secret bits) at the expense of $\sim 100$ (a few hundred) entangled pairs, i.e., the number of secret bits gained per use of an entangled pair is roughly 0.1 or less. The efficiency of the proposed scheme is thus somewhat lower than that of the entanglement-based cryptographic scheme(E91)[9].

We note that the proposed protocol can be used for Alice and Bob to send secretly to each other directions in space and consequently to possess two private shared reference frames. They need of course to store the information on their Euler angles in the constants $a_{0}, a_{1}$ and $b_{0}, b_{1}$, respectively. Shared reference frames are a resource for quantum communications $[17,18,19]$. A standard protocol[18] to send directions in space when Alice and Bob share entangled 
pairs, say in $\left(\Phi_{00}\right)_{A B}$, requires Alice to perform a projection measurement in her $|0\rangle-|1\rangle$ basis on the particle $A$ of each entangled pair and announce publicly the outcome of each measurement. Bob would then follow with his projection measurement in his $|0\rangle-|1\rangle$ basis on the corresponding particle B of each entangled pair. If a sufficiently large number $N \gg 1$ of projection measurements are performed by Alice and Bob, the number $N_{s}$ of times that Alice and Bob obtain the same measurement outcome will be given by $\frac{N_{s}}{N}=\cos ^{2} \theta$, where $\theta$ is the angle between Alice's and Bob's axes.[ We assume that qubits

are polarized photons. If we take spins for qubits, then $\left.\frac{N_{s}}{N}=\cos ^{2} \frac{\theta}{2}\right]$. Provided that Alice and Bob perform each of their projection measurements individually, essentially the same statistical analysis based on Eq.(9) applies here, and thus the efficiency of our proposed protocol is of the same order of magnitude as that of this standard entanglement-based protocol. [If, however, we allow Bob to make collective measurements in the standard protocol, the efficiency can be higher. See, for example, Ref.[20].] Our protocol, however, requires both Alice and Bob to perform Bell-state measurements, which are in general more difficult to perform than von-Neumann projection measurements. One advantage of our protocol is that it is symmetric with respect to Alice and Bob and allows both Alice and Bob to gain information, whereas in standard schemes the information usually flows one way.

\section{Generalization to higher-dimensional systems}

We now consider a generalization of the above scheme to higher-dimensional systems, i.e., to "qudits". The generalized Bell states for a qudit can be defined as $[21,22]$

$$
\left|\Phi_{i j}\right\rangle_{A B}=\frac{1}{\sqrt{d}} \sum_{q=0}^{d-1} \omega^{j q}|q\rangle_{A}|q+i\rangle_{B} ; i, j=0,1, \ldots, d-1
$$


where $\omega=e^{i \frac{2 \pi}{d}}$. Let us assume that Alice and Bob share a large number $\mathrm{N}(\gg 1)$ of entangled pairs $A$ 's and $B$ 's in the generalized Bell state $\left|\Phi_{00}\right\rangle_{A B}$. Alice performs a series of $\mathrm{N}$ Bell-state measurements on pairs of qudit $A$ and another qudit $\alpha$ she prepared in state $|\psi\rangle_{\alpha}=\sum_{i=0}^{d-1} a_{i}|i\rangle_{\alpha}$, while Bob performs a series of N Bell-state measurements on pairs of qudit $B$ and yet another qudit $\beta$ he prepared in state $|\psi\rangle_{\beta}=\sum_{i=0}^{d-1} b_{i}|i\rangle_{\beta}$. As in the qubit case, the total wave function $|\psi\rangle_{\alpha \beta A B}$ can be expanded in terms of the products of the generalized Bell states $\left|\Phi_{i_{1} j_{1}}\right\rangle_{\alpha A}\left|\Phi_{i_{2} j_{2}}\right\rangle_{\beta B}$ as

$$
|\psi\rangle_{\alpha \beta A B}=\sum_{i_{1}, j_{1}, i_{2}, j_{2}=0}^{d-1}\left|\Phi_{i_{1} j_{1}}\right\rangle_{\alpha A}\left|\Phi_{i_{2} j_{2}}\right\rangle_{\beta B} V_{i_{1} j_{1} i_{2} j_{2}}
$$

where $V_{i_{1} j_{1} i_{2} j_{2}}$ is given by

$$
V_{i_{1} j_{1} i_{2} j_{2}}=\frac{1}{d \sqrt{d}} \omega^{\left(i_{1} j_{1}+i_{2} j_{2}\right)} \sum_{m=0}^{d-1} \omega^{-\left(j_{1}+j_{2}\right) m} a_{m-i_{1}} b_{m-i_{2}}
$$

where all indices are now evaluated modulo d. Eq.(13) indicates that the probabilities $P_{i_{1} j_{1} i_{2} j_{2}}=\left|V_{i_{1} j_{1} i_{2} j_{2}}\right|^{2}$ take on the same value if $i_{1}-i_{2}(\bmod \mathrm{d})$ is the same and if $j_{1}+j_{2}(\bmod \mathrm{d})$ is the same. Thus, there are $d^{2}$ different values of the probabilities $P_{i_{1} j_{1} i_{2} j_{2}}$ 's. Eq.(13) then provides $\left(d^{2}-1\right)$ independent equations that relate the constants, $a_{0}, a_{1}, \ldots, a_{d-1} ; b_{0}, b_{1}, \ldots, b_{d-1}$. To any third party other than Alice and Bob, the number of unknowns contained in these constants is $(4 d-4)$. There are , however, only $(2 d-2)$ unknowns, as far as Alice or Bob is concerned. By agreeing to publicly announce their measurement results only when the outcome is among judiciously chosen generalized Bell states, Alice and Bob can limit the number of probabilities that can be determined experimentally in such a way that the number of equations that relate the experimentally determined probabilities with the constants $a_{i}$ 's and $b_{i}$ 's is greater than or equal to $(2 d-2)$ but less that $(4 d-4)$. This way, Alice and Bob can send secret messages in the form of $(2 d-2)$ constants to each other and as a result secretely share $(4 d-4)$ constants between them. 
As an example consider the case $d=3$. If Alice and Bob announce results of the Bell-state measurements only when they measure either $\Phi_{00}$ or $\Phi_{21}$, they can determine experimentally the four probabilities $P_{0000}^{e x p}, P_{2121}^{e x p}, P_{0021}^{e x p}$ and $P_{2100}^{e x p}$, which are given by

$$
\begin{aligned}
& P_{0000}=\frac{1}{27}\left|a_{0} b_{0}+a_{1} b_{1}+a_{2} b_{2}\right|^{2} \\
& P_{2121}=\frac{1}{27}\left|a_{0} b_{0}+a_{1} b_{1} \omega+a_{2} b_{2} \omega^{2}\right|^{2} \\
& P_{0021}=\frac{1}{27}\left|a_{2} b_{0}+a_{0} b_{1} \omega^{2}+a_{1} b_{2} \omega\right|^{2} \\
& P_{2100}=\frac{1}{27}\left|a_{1} b_{0} \omega^{2}+a_{2} b_{1} \omega+a_{0} b_{2}\right|^{2}
\end{aligned}
$$

where $\omega=e^{i \frac{2 \pi}{3}}$. Eqs. (14a)-(14d) are sufficient for Alice and Bob to determine their four unknowns, allowing them to exchange messages in the form of four constants each.

\section{Generalization to multipartite entanglement}

Another possible generalization of the proposed scheme is to the case of multiparty communications. Let us consider the case when $\mathrm{N}(>2)$ parties share an N-qubit entangled state of Greenberger-Horne-Zeilinger (GHZ) type [23] given by

$$
|\Phi\rangle_{A B \ldots N}=\frac{1}{\sqrt{2}}\left(|0\rangle_{A}|0\rangle_{B} \ldots|0\rangle_{N}+|1\rangle_{A}|1\rangle_{B} \ldots|1\rangle_{N}\right)
$$

where the letter $\mathrm{N}$ (also the small letter $n$ and the Greek letter $\nu$ ) refers to the Nth party or Nth qubit. Each party has, in addition to the qubit K of Eq.(15) [ the letter $\mathrm{K}$ (and also the small letter $\mathrm{k}$ and the Greek letter $\kappa$ ) denotes the Kth party or Kth qubit, where $1 \leq K \leq N]$, another qubit $\kappa$ which she or he 
prepared in state

$$
|\psi\rangle_{\kappa}=k_{0}|0\rangle_{\kappa}+k_{1}|1\rangle_{\kappa}
$$

Each party then performs a Bell-state measurement upon the qubits $\kappa$ and $\mathrm{K}$. The total wave function for the $2 \mathrm{~N}$ qubits $\alpha, \beta, \ldots \nu, A, B, \ldots N$ can be expanded as

$|\psi\rangle_{\alpha, \beta, \ldots \nu, A, B, \ldots N}=\sum_{i_{1}, j_{1}, i_{2}, j_{2}, \ldots, i_{N}, j_{N}=0}^{1}\left(\Phi_{i_{1} j_{1}}\right)_{\alpha A}\left(\Phi_{i_{2} j_{2}}\right)_{\beta B} \ldots\left(\Phi_{i_{N} j_{N}}\right)_{\nu N} V_{i_{1} j_{1} i_{2} j_{2} \ldots i_{N} j_{N}}$

A straightforward algebra yields

$$
\begin{aligned}
& V_{i_{1} j_{1} i_{2} j_{2} \ldots i_{N} j_{N}}= \\
& \frac{1}{(\sqrt{2})^{N+1}}(-1)^{i_{1} j_{1}+i_{2} j_{2}+\cdots+i_{N} j_{N}}\left[a_{i_{1}} b_{i_{2}} \ldots n_{i_{N}}+(-1)^{j_{1}+j_{2}+\cdots+j_{N}} a_{i_{1}+1} b_{i_{2}+1} \ldots n_{i_{N}+1}\right]
\end{aligned}
$$

In Eq.(18), the constants $n_{0}$ and $n_{1}$ define the state of the qubit $\nu$ in which the Nth party prepared according to

$$
|\psi\rangle_{\nu}=n_{0}|0\rangle_{\nu}+n_{1}|1\rangle_{\nu}
$$

Eq.(18) indicates that there are $2^{N}$ different values for the probabilities $P_{i_{1} j_{1} i_{2} j_{2} \ldots i_{N} j_{N}}$ $=\left|V_{i_{1} j_{1} i_{2} j_{2} \ldots i_{N} j_{N}}\right|^{2}$. To any member of the $N$ parties sharing the entanglement of Eq.(15), the number of unknowns is $(2 N-2)$, while it is $(2 N)$ to any outsider. As before, by agreeing to publicly announce the measurement results only when the outcome is among judiciously chosen Bell states, each of the $\mathrm{N}$ parties can secretely send his message in the form of two constants to all others of the $\mathrm{N}$ parties, so that the $\mathrm{N}$ parties can share secretely the messages in the form of $2 N$ constants.

As an example, consider the case $N=3$. Let us assume that the three parties, Alice, Bob and Charlie agree that Charlie is the last one to make an announce- 
ment each time, that Alice and Bob announce the measurement results only when they obtain either $\Phi_{10}$ or $\Phi_{11}$, and that Charlie announces his measurement result only when both Alice and Bob announce their measurement results and he(Charlie) obtains either $\Phi_{10}$ or $\Phi_{11}$ or $\Phi_{00}$. The probabilities that can be determined experimentally are then the following:

$$
\begin{aligned}
& P_{101010}^{\exp }=P_{101111}^{\exp }=P_{111110}^{\exp }=P_{111011}^{\exp }=\frac{1}{16}\left|a_{0} b_{0} c_{0}+a_{1} b_{1} c_{1}\right|^{2} \\
& P_{111010}^{\exp }=P_{101110}^{\exp }=P_{101011}^{\exp }=P_{111111}^{\exp }=\frac{1}{16}\left|a_{0} b_{0} c_{0}-a_{1} b_{1} c_{1}\right|^{2} \\
& P_{101100}^{\exp }=P_{111000}^{\exp }=\frac{1}{16}\left|a_{0} b_{0} c_{1}-a_{1} b_{1} c_{0}\right|^{2} \\
& P_{101000}^{\exp }=P_{111100}^{\exp }=\frac{1}{16}\left|a_{0} b_{0} c_{1}+a_{1} b_{1} c_{0}\right|^{2}
\end{aligned}
$$

Since each of Alice, Bob and Charlie has four unknowns, she or he can use Eqs. (20a)-(20d) to solve for her or his unknowns. This allows Alice, Bob and Charlie to share secretely six constants among them.

If Alice, Bob and Charlie are limited to linear-optical Bell-state measurements, they can only determine eight probabilities $P_{1 j_{1} 1 j_{2} 1 j_{3}}\left(j_{1}, j_{2}, j_{3}=0,1\right)$ of Eqs. (20a) and (20b) experimentally. In this situation, Alice, Bob and Charlie each needs to announce publicly one of the constants, say $a_{0}, b_{0}$ and $c_{0}$. Each of Alice, Bob and Charlie then has two unknowns for which Eqs.(20a) and (20b) provide sufficient information. In this case, however, the number of constants that the three parties can secretely share is reduced to three.

\section{Case of no shared reference frames}

So far, we have assumed that Alice and Bob have exactly the same basis for the states $|0\rangle$ and $|1\rangle$. Thus, if the qubits we consider are polarized photons or spins, we assume that Alice and Bob share a spatial reference frame. As far as our proposed protocol is concerned, this shared reference frame does not need 


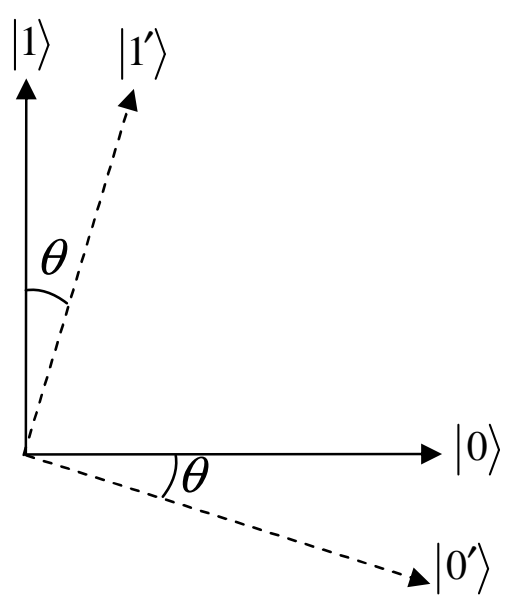

Fig. 2. Alice's reference frame $(|1\rangle$ and $|0\rangle)$ and Bob's reference frame $\left(\left|1^{\prime}\right\rangle\right.$ and $\left.\left|0^{\prime}\right\rangle\right)$.

to be private, because the privacy of the protocol does not depend upon the privacy of the reference frame. The shared reference frame can, for example, be a specific direction with respect to a fixed star.

It may happen, however, that Alice and Bob, for reasons of better security, want to use reference frames of their own choice which may not coincide, or that their reference frames are inadvertently misaligned. As show below, our proposed protocol still works, even if Alice's reference frame does not coincide with Bob's. Let us suppose that Bob's axis makes an angle $\theta$ with respect to Alice's, as shown in Fig. 2. The initial state for the four particles $\alpha, \beta, A$ and $B$ are now written as

$$
|\Psi\rangle_{\alpha \beta A B}=\left(a_{0}|0\rangle_{\alpha}+a_{1}|1\rangle_{\alpha}\right) \frac{1}{\sqrt{2}}\left(|0\rangle_{A}|0\rangle_{B}+|1\rangle_{A}|1\rangle_{B}\right)\left(b_{0}\left|0^{\prime}\right\rangle_{\beta}+b_{1}\left|1^{\prime}\right\rangle_{\beta}\right)
$$

where $|0\rangle$ and $|1\rangle$ denote Alice's basis states and $\left|0^{\prime}\right\rangle$ and $\left|1^{\prime}\right\rangle$ Bob's basis states. We assume that Alice prepares the entangled pair $A$ and $B$ in $\left|\Phi_{00}\right\rangle_{A B}$, keeps $A$ and sends $B$ to Bob. Now Alice performs her Bell state measurement on the pair $\alpha A$ in her $|0\rangle-|1\rangle$ basis, whereas Bob performs his Bell state measurement on the pair $\beta B$ in his $\left|0^{\prime}\right\rangle-\left|1^{\prime}\right\rangle$ basis. We thus need to express $|0\rangle_{B}$ and $|1\rangle_{B}$ 
in terms of $\left|0^{\prime}\right\rangle_{B}$ and $\left|1^{\prime}\right\rangle_{B}$, and expand the wave function $|\Psi\rangle_{\alpha \beta A B}$ as

$$
|\Psi\rangle_{\alpha \beta A B}=\sum_{i_{1}, j_{1}, i_{2}, j_{2}=0}^{1}\left|\Phi_{i_{1} j_{1}}\right\rangle_{\alpha A}\left|\Phi_{i_{2} j_{2}}^{\prime}\right\rangle_{\beta B} V_{i_{1} j_{1} i_{2} j_{2}}
$$

where $\left|\Phi_{i j}^{\prime}\right\rangle$ refers to Bell states of Eq. (1) defined in terms of Bob's basis $\left|0^{\prime}\right\rangle$ and $\left|1^{\prime}\right\rangle$. A straightforward algebra yields, for the probabilities $P_{i_{1} j_{1} i_{2} j_{2}}=$ $\left|V_{i_{1} j_{1} i_{2} j_{2}}\right|^{2}$,

$$
P_{0000}=P_{1111}=\frac{1}{8}\left|a_{0} b_{0} \cos \theta-a_{1} b_{0} \sin \theta+a_{0} b_{1} \sin \theta+a_{1} b_{1} \cos \theta\right|^{2} \equiv \frac{1}{2} P_{1}
$$

$$
P_{0001}=P_{1110}=\frac{1}{8}\left|a_{0} b_{0} \cos \theta-a_{1} b_{0} \sin \theta-a_{0} b_{1} \sin \theta-a_{1} b_{1} \cos \theta\right|^{2} \equiv \frac{1}{2} P_{2}
$$

$$
\begin{aligned}
& P_{0010}=P_{1101}=\frac{1}{8}\left|a_{0} b_{0} \sin \theta+a_{1} b_{0} \cos \theta+a_{0} b_{1} \cos \theta-a_{1} b_{1} \sin \theta\right|^{2} \equiv \frac{1}{2} P_{3} \\
& P_{0011}=P_{1100}=\frac{1}{8}\left|a_{0} b_{0} \sin \theta+a_{1} b_{0} \cos \theta-a_{0} b_{1} \cos \theta+a_{1} b_{1} \sin \theta\right|^{2} \equiv \frac{1}{2} P_{4}
\end{aligned}
$$

$$
P_{0100}=P_{1011}=\frac{1}{8}\left|a_{0} b_{0} \cos \theta+a_{1} b_{0} \sin \theta+a_{0} b_{1} \sin \theta-a_{1} b_{1} \cos \theta\right|^{2} \equiv \frac{1}{2} P_{5}
$$

$$
P_{0101}=P_{1010}=\frac{1}{8}\left|a_{0} b_{0} \cos \theta+a_{1} b_{0} \sin \theta-a_{0} b_{1} \sin \theta+a_{1} b_{1} \cos \theta\right|^{2} \equiv \frac{1}{2} P_{6}
$$




$$
\begin{aligned}
& P_{0110}=P_{1001}=\frac{1}{8}\left|a_{0} b_{0} \sin \theta-a_{1} b_{0} \cos \theta+a_{0} b_{1} \cos \theta+a_{1} b_{1} \sin \theta\right|^{2} \equiv \frac{1}{2} P_{7} \\
& P_{0111}=P_{1000}=\frac{1}{8}\left|a_{0} b_{0} \sin \theta-a_{1} b_{0} \cos \theta-a_{0} b_{1} \cos \theta-a_{1} b_{1} \sin \theta\right|^{2} \equiv \frac{1}{2} P_{8}
\end{aligned}
$$

where the probabilities are further restricted by the identities

$$
\begin{gathered}
P_{1}+P_{2}+P_{3}+P_{4}=\frac{1}{2} \\
P_{2}+P_{3}=P_{5}+P_{8} \\
P_{1}+P_{4}=P_{6}+P_{7}
\end{gathered}
$$

Comparison of Eqs.(23) with Eqs.(7) indicates that the misalignment of Bob's axis with respect to Alice's axis partly breaks degeneracies among the probabilities. For example, $P_{1111}$ is no longer equal to $P_{1010}$, and $P_{1110}$ is no longer equal to $P_{1011}$. The difference between these probabilities can thus be considered as a measure of the misalignment.

Our scheme for quantum message exchange can proceed exactly as before. We let Alice and Bob announce publicly their measurement results only when the outcome is either $\Phi_{10}$ or $\Phi_{11}$. The four experimentally determined probabilities $P_{1010}^{e x p}, P_{1111}^{e x p}, P_{1011}^{e x p}$, and $P_{1110}^{e x p}$ then provide four equations, Eqs. (23a), (23b), (23e) and (23f), that relate the five constants; $a_{0}, a_{1}, b_{0}, b_{1}$ and the angle $\theta$. To Alice(Bob) there are three unknowns $b_{0}, b_{1}\left(a_{0}, a_{1}\right)$ and $\theta$, whereas to any third party the number of unknowns is five. Only Alice and Bob can thus determine the five constants $a_{0}, a_{1}, b_{0}, b_{1}$ and $\theta$. Our scheme thus allows Alice and Bob 
not only to secretely share the four constants but also to determine the angle between their reference frames.

\section{$7 \quad$ Summary and discussion}

We have analyzed a situation in which each of two (or more) parties sharing entanglement performs a Bell-state measurement upon the entangled particle in his (or her) possession and another particle he(or she) prepared in a specific state. The probability for a joint measurement outcome corresponding to a given combination of the Bell states depends critically upon the states of the particles involved. Taking advantage of the fact that each person belonging to the parties sharing entanglement and only he(or she) knows the state of the particle he(or she) prepared, we suggest a scheme by which two(or more) parties sharing entanglement can secretely and simultaneously exchange messages. For the case of two parties sharing entangled qubits, the scheme requires Bell-state measurements that distinguish only two out of the four Bell states, which can be accomplished using only linear optical devices. The scheme thus provides an experimentally feasible means of two-way communication.

We should emphasize that, although it may not be apparent at first sight, entanglement plays a critical role in the proposed scheme. It is through entanglement that the joint probabilities appear in an "entangled" form as give in Eqs.(7) and that the separate probabilities for either Alice or Bob to obtain any arbitrary Bell state are evenly distributed regardless of which Bell state we consider. Information on the constants $a_{0}, a_{1}, b_{0}$ and $b_{1}$ can be obtained only by looking at the joint probabilities. On the other hand, if the qubits $\mathrm{A}$ and B were not entangled, Alice's Bell-state measurements would be completely independent of Bob's Bell-state measurements, and information on the constants $a_{0}$ and $a_{1}\left(b_{0}\right.$ and $\left.b_{1}\right)$ would be obtained by looking only at the results of Alice's (Bob's) Bell-state measurements. Bob(Alice) would need as much 
information as any third party in order to determine $a_{0}$ and $a_{1}\left(b_{0}\right.$ and $\left.b_{1}\right)$ from the results of Alice's (Bob's) Bell-state measurements. The parties sharing entanglement have advantages only because the joint probabilities for Alice's and Bob's Bell-state measurements are "entangled". Of course, the maximal advantage is provided by the maximal entanglement which we have assumed. In general, as the degree of shared entanglement is decreased, the joint probabilities exhibit less degree of entanglement, and as a result the parties sharing entanglement has less degree of advantage over a third party.

\section{Acknowledgements}

This research was supported by a Grant from the Korea Science and Engineering Foundation(KOSEF) through Korea-China International Cooperative Research Program and from the Ministry of Science and Technology(MOST) of Korea. The authors thank Professor Gui Lu Long of Tsinghua university of China for helpful discussions.

\section{References}

[1] C. H. Bennett and S. J. Wiesner, Phys. Rev. Lett. 69 (1992) 2881.

[2] K.Mattle, H.Weinfurter, P.G.Kwiat, and A. Zeilinger, Phys. Rev. Lett. 76 (1996) 4656.

[3] C.H.Benett, G. Brassard, C. Crepeau, R. Jozsa, A. Peres, and W. K. Wootters, Phys. Rev. Lett. 70 (1993) 1895.

[4] D. Boschi, S. Branca, F. De Martini, L. Hardy, and S. Popescu, Phys. Rev. Lett. 80 (1998) 1121.

[5] D. Bouwmeester, J.W. Pan, K. Mattle, M. Eibl, H. Weinfurter, and A. Zeilinger, Nature (London) 390 (1997) 575.

[6] A. Furusawa, J. L. Sørensen, S.L.Braustein, C. A. Fuchs, H. J. Kimble, and E. S. Polzik, Science 282 (1998) 706.

[7] H.W.Lee and J. Kim, Phys. Rev. A 63 (2001) 012305. 
[8] E. Lombardi, F. Sciarrino, S. Popescu, and F. De Martini, Phys. Rev. Lett. 88 (2002) 070402.

[9] A. K. Ekert, Phys. Rev. Lett. 67 (1991) 661.

[10] T. Jennewein, C. Simon, G. Weihs, H. Weinfurter, and A. Zeilinger, Phys. Rev. Lett. 84 (2000) 4729;

D. S. Naik, C. G. Peterson, A. G. White, A. J. Berglund, and P. G. Kwiat, Phys. Rev. Lett. 84 (2000) 4733;

W. Tittel, J. Brendel, H. Zbinden, and N. Gisin, Phys. Rev. Lett. 84 (2000) 4737 .

[11] J.W.Lee, E.K.Lee, Y.W.Chung, H.W.Lee, and J.Kim, Phys. Rev. A 68 (2003) 012324.

[12] R. Raussendorf and H.J.Briegel, Phys. Rev. Lett. 86 (2001) 5188.

[13] M.A.Nielsen, quant-ph/0402005.

[14] N.Lütkenhaus, J.Calsamiglia, and S.A.Suominen, Phys. Rev. A 59 (1999) 3295.

[15] B.A.Nguyen, Phys. Lett. A 328 (2004) 6.

[16] See, for example, F. Reif, Fundamentals of Statistical and Thermal Physics (McGraw-Hill, New York, 1965), Ch. 1.

[17] S.J.van Enk, quant-ph/0410083

[18] T.Rudolph and L.Grover, Phys.Rev.Lett. 91, 217905(2003).

[19] S.D.Bartlett, T.Rudolph, and R.W.Spekkens, Phys.Rev.A 70, 032307 (2004).

[20] S.Massar and S.Popescu, Phys.Rev.Lett. 74, 1259(1995);

R.Derka, V.Buzek, and A.K.Ekert, Phys.Rev.Lett. 80, 1571 (1998);

N.Gisin and S.Popescu, Phys.Rev.Lett. 83, 432 (1999);

S.Massar, Phys.Rev.A 62, 040101(R) (2000);

A.Peres and P.F.Scudo, Phys.Rev.Lett. 86, 4160 (2001).

[21] G.Alber, A.Delgado, N.Gisin, and I.Jex, quant-ph/0008022

[22] S.J. van Enk, Phys. Rev. Lett. 91 (2003) 017902.

[23] D.M.Greenberger, M.A.Horne, A.Shimony, and A.Zeilinger, Amer.J.Phys. 58 (1990) 1131;

N.D.Mermin, Phys. Today 43 (1990) 9. 